\title{
Management of Local Soft Tissue Recurrent Osteosarcoma After Wide Resection and Reconstruction with Megaprosthesis: A Case Report
}

\author{
Venansius Herry Perdana Suryanta, Muhammad Naseh Sajadi Budi \\ Department of Orthopaedic and Traumatology, Faculty of Medicine Universitas Padjadjaran/Hasan Sadikin General \\ Hospital, Bandung, Indonesia
}

\section{ARTICLE INFO}

Received : 25 July 2019

Reviewed : 15 August 2019

Accepted : 20 November 2019

Keywords:

biopsy, local recurrence, osteosarcoma

\begin{abstract}
Introduction: Limb salvage surgery involves all of the surgical methods to achieve the eradication of a malignant neoplasm and restoration of the limb with a satisfactory oncologic, functional, and cosmetic outcome. Rates of local recurrence are $4 \%$ to $10 \%$. There is a chance of local recurrence from the contamination of biopsy path. Neoadjuvant chemotherapy has a guarding effect on managing neoplasm infiltration at the biopsy location.
\end{abstract}

Case Presentation: An 18 year old male experienced a new painless lump at his right anterior thigh with size about $5 \mathrm{~cm}$ in diameter since 5 months ago. Previously, he underwent limb salvage surgery with megaprosthesis about 6 months ago due to osteosarcoma at his right distal femur. He also got neoadjuvant and adjuvant chemotherapy for 6 cycles. Mass removal was done and $1.5 \mathrm{~cm}$ mass in diameter was found within quadriceps muscle with a soft consistency and well-defined border from the previous biopsy site that had not been resected. The specimen result was osteosarcoma surrounded by tumor-free tissue. Postoperatively, he still had the same range of motion function as before.

Conclusions: We need to consider the previously contaminated biopsy path that could lead to local recurrence. Factors that affect the prediction of the recurrent disease are the disease-free time period, location of recurrence and histological response to therapy and the capability to achieve total surgical removal. Tumor removal followed by the local radiation and chemotherapy is the preferred treatment for recurrence.

\section{INTRODUCTION}

The combination of clinical, laboratory, radiographic and histological features is required concerning the approach to neoplasms of the musculoskeletal system for correct diagnosis and management leading to successful therapy. In this view, the biopsy is shown out as a significant step, is crucial for the definitive diagnosis and to recognize the histological pattern of neoplasm [1]. A biopsy must allow sufficient and representative tissue specimens for the correct diagnosis. Excessive manipulation of the lesion should be avoided because it could alter the tumor involvement between anatomical compartments and the contamination of surrounding tissues with tumor cells [2].

Removal of the biopsy path at the moment of tumor surgical resection is promoted by most surgeons in the treatment of musculoskeletal neoplasms, explaining that this path is likely contaminated by tumor cells. Though no evidence for this method has been found in any scientific investigations, and it is based more on individual experience than on the current research [3].

There is a lack of specific investigations on biopsy paths in samples of musculoskeletal neoplasms in the literature [2]. In orthopedic oncology, biopsy technique improvement may be provided by understanding the characteristics of contamination biopsy paths. The purpose of this study is to report a patient with local soft tissue recurrent osteosarcoma after wide resection and reconstruction with megaprosthesis and its management.

\section{CASE PRESENTATION}

An 18 year old male experienced a new painless lump at his right thigh at anterior aspect with size about $5 \mathrm{~cm}$ in diameter since 5 months before. The lump had a soft consistency and well-defined border (Figure 1). He never experienced pain or difficulty when walking. 


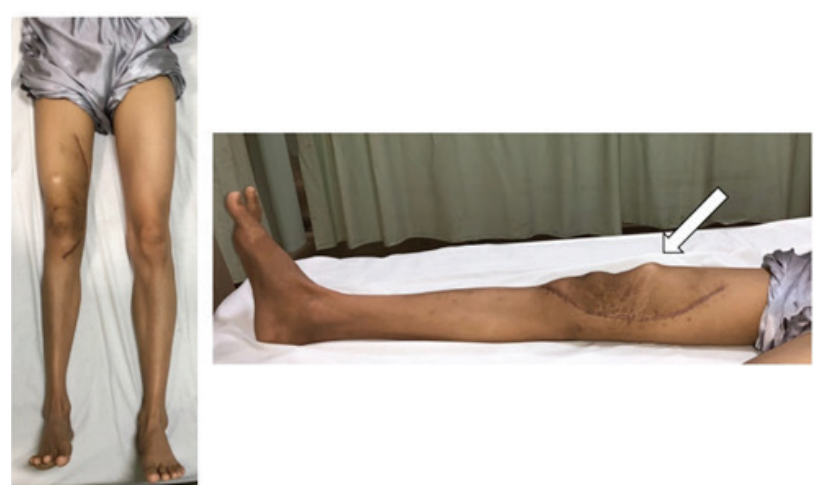

Figure 1. Clinical appearance of the tumor

He had a previous history of limb salvage surgery with megaprosthesis about 6 months ago due to osteosarcoma at his right distal femur without metastasis to the lung (Figure 2). He also got 3 cycles neoadjuvant (preoperative) chemotherapy and 3 cycles adjuvant (postoperative) chemotherapy for local control and prevention of metastases with methotrexate, doxorubicin, and cisplatin. The previous surgeon (orthopedic resident) had a core biopsy before at the anterior side of distal femur and the biopsy path was not resected when the limb salvage surgery was done. There was a high chance that the lump arose from the previous biopsy path by the other surgeon.

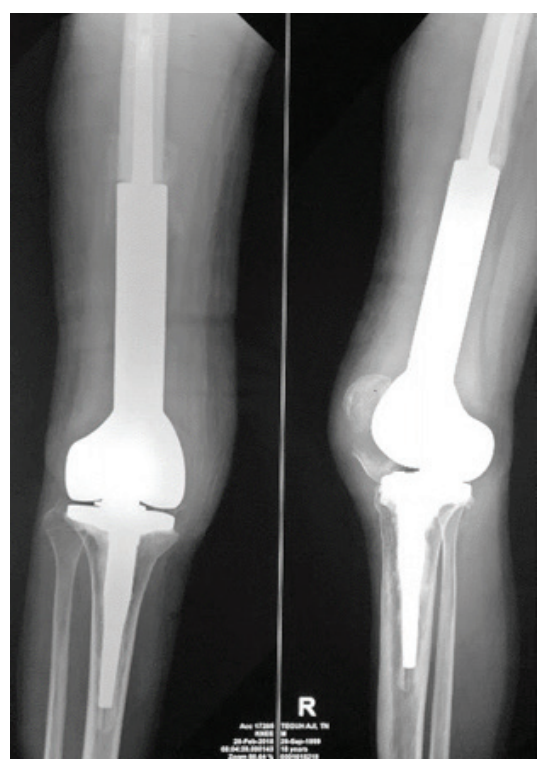

Figure 2. X-ray of the local soft tissue recurrence

Intraoperatively, we did the wide resection of the quadriceps muscle. A mass was found with a size of $1.5 \mathrm{~cm}$ in diameter within quadriceps muscle with soft consistency and well-defined border from the previous biopsy incision (Figure 3 ). The biopsied result was osteosarcoma surrounded by tumor-free tissue. Postoperatively, he still had the same range of motion function as before.
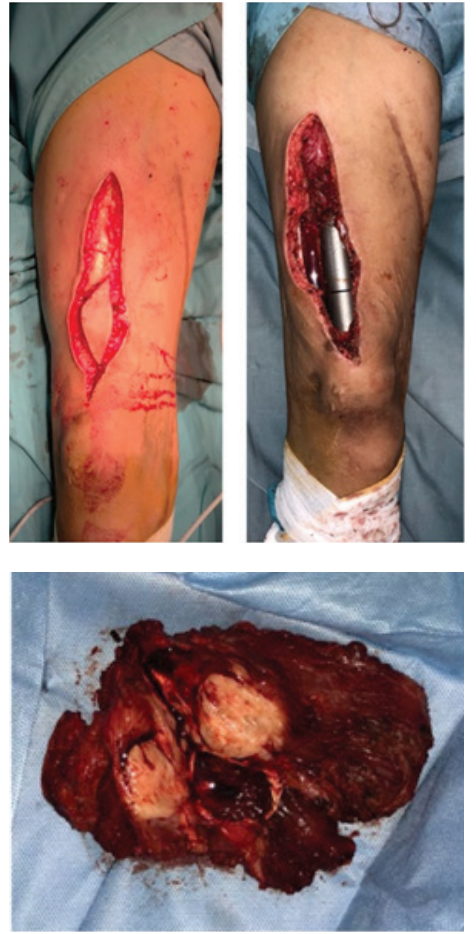

Figure 3. Intraoperative finding with the mass inside quadriceps muscle.

\section{DISCUSSION}

In our case, the previous surgeon (orthopedic resident) did the biopsy at the anterior side of the femur because of the previous mass was easily palpated and more prominent at the anterior, however the other surgeon (orthopedic oncology specialist) who did the limb salvage surgery made incision at the medial side and the previous biopsy path had not been resected because it was disguised with healthy skin and the surgeon missed it. Liu et al. [4] suggest that the surgical method may have to be changed to add the possibly contaminated tissue in the resection if a core needle bone biopsy were to reach through tissues outside the intended incision plane. The patient had less attractive options that are either a wider irradiation area (if the tumor is radiosensitive) or a higher risk of local tumor recurrence at the biopsy path [4].

Cannon and Dyson reinforced the opinion inside the orthopedic population that the biopsy path in cases of musculoskeletal neoplasms might be contaminated by tumor cells. The appearance of local tumor recurrence was statistically lower in cases with resected biopsy path [5]. The research related to this issue shows that in cases with the biopsy path had not been resected, local recurrence was constantly seen [6-8]. Besides that, Kaffenberger et al. [8] and Saghieh et al. [9] mentioned that no local recurrence was seen in which biopsy paths created using the percutaneous method that had not been resected. 
Several concerns are reported in a broad type of studies, and untested hypotheses have appeared. Between these, there is a hypothesis that attempts to get some tissue specimens in biopsies are correlated with higher distribution and a consequently greater possibility of contamination of the biopsy path. Another hypothesis that has been announced is that biopsies done using a percutaneous method are correlated with smaller contamination of the biopsy path because they comprise more limited manipulation of the tumor tissue. It was furthermore observed that contamination of the biopsy path happens more often in cases of soft-tissue sarcoma than in bone and cartilage tumors [2].

For the past 10 years, adjuvant methods have hugely affected the therapy of neoplasms of the musculoskeletal system. Chemotherapy is an effective method in the treatment of some bone tumors, especially Ewing's sarcoma and osteosarcoma, which became more beneficial after the introduction of this therapeutic modality, considering for a historic change in the prognosis of these neoplasms. Administration of the neoadjuvant chemotherapy before surgical resection of the tumor intends to provoke tumor regression, decrease tumor extent at surgery, and provide a surgical treatment with lower functional impairment. Some authors think that chemotherapy has a protective effect on the biopsy site for control of tumor infiltration [2].

Local recurrence should be managed by local surgical management that needs to be specified to individual anatomy, especially the nearness to vital structures and availability of adequately wide margins. Wide resection of the locally recurrent tumor with margins of more than $1 \mathrm{~cm}$ was significantly associated with a longer Post-Recurrence Survival compared to positive margins or below $1 \mathrm{~cm}$. For local control of recurrent tumors is essential to get adequate wide resection [10].

Amputations done for first local recurrence did not seem to produce a significant survival advantage. Patients that get local recurrence without metastasis could be treated with neoadjuvant chemotherapy and suggested for local resection and adjuvant chemotherapy [11].

\section{CONCLUSIONS}

This case report showed that biopsy path contamination may lead to the local recurrence even with the percutaneous technique and neoadjuvant therapy, confirming the necessity for biopsy path removal at the time of tumor resection. Besides, biopsies should be done by the same surgeon that will do the surgical operation of the tumor and performed in the center of oncology surgery. Tumor removal combined with neoadjuvant chemotherapy is the preferred treatment for recurrences.

\section{DECLARATIONS}

\section{Competing of Interest}

The authors declare no competing interest in this study.

\section{Acknowledgement}

The Author(s) wishes to thank all oncology orthopedic staffs: Professor Darmadji and Herry Herman,dr.

\section{REFERENCES}

1. Ilaslan H, Schils J, Nageotte W, Lietman SA, Sundaram M. Clinical presentation and imaging of bone and softtissue sarcomas. Cleve Clin J Med. 2010;77(Suppl):S2S7

2. Oliveira MP, Lima PM, da Silva HJ, de Mello RJ. Neoplasm seeding in biopsy tract of the musculoskeletal system: A systematic review. Acta Ortop Bras. 2014;22(2):106-10.

3. Oliveira MP, Lima PM, de Mello RJ. Tumor contamination in the biopsy path of primary malignant bone tumors. Rev Bras Ortop. 2015;47(5):631-37.

4. Liu PT, Valadez SD, Chivers S. Anatomically based guidelines for core needle biopsy of bone tumors: Implications for limb-sparing surgery. Radiographics. 2007;27(1):189-205.

5. Cannon SR, Dyson PHP. Relationship of the site of open biopsy of malignant bone tumours to local recurrence following resection and prosthetic replacement. J Bone Joint Surg Br. 1987;69:492.

6. Davies NM, Livesley PJ, Cannon SR. Recurrence of an osteosarcoma in a needle biopsy track. J Bone Joint Surg Br. 1993;75(6):977-8.

7. Mohana R, Faisham W, Zulmi W, Nawfar AS, Effat $\mathrm{O}$, Salzihan MS. The incidence of malignant infiltration in the biopsy tract of osteosarcoma. Malays Orthop J. 2007;1:7-10.

8. Kaffenberger BH, Wakely PE, Jr, Mayerson JL. Local recurrence rate of fine-needle aspiration biopsy in primary high-grade sarcomas. J Surg Oncol. 2010;101(7):618-21

9. Saghieh S, Masrouha KZ, Musallam KM, et al. The risk of local recurrence along the core-needle biopsy tract in patients with bone sarcomas. lowa Orthop J. 2010;30:80-3.

10. Loh AH, Navid F, Wang C, et al. Management of local recurrence of pediatric osteosarcoma following limb-sparing surgery. Ann Surg Oncol. 2014;21(6):1948-55.

11. Nathan SS, Gorlick R, Bukata S. Treatment algorithm for locally recurrent osteosarcoma based on local disease-free interval and the presence of lung metastasis. Cancer. 2006;107:1607-16. 